\title{
La neuroscience éducationnelle : enrichir la recherche en éducation par l'ajout de méthodes psychophysiologiques pour mieux comprendre l'apprentissage
}

https://doi.org/10.24046/neuroed.20120101.115

\author{
Stephen R. CAMPBELL ${ }^{1}$ et Pierre PAGÉ2, * \\ ${ }^{1}$ Laboratoire de neuroscience (ENGRAMMETRON), Faculté d'éducation, Université Simon Fraser, \\ Vancouver \\ ${ }^{2}$ Faculté des sciences de l'éducation, Université Laval, Québec \\ ${ }^{*}$ Courriel: pierre.page@fse.ulaval.ca
}

\begin{abstract}
Résumé
L'objet de cet article consiste à présenter des arguments en faveur de la recherche en neuroscience éducationnelle qui peut être bien davantage qu'un simple domaine d'application des neurosciences cognitive et sociale (Campbell, 2011). L'argumentaire s'arrime d'abord autour de la présentation des éléments du cadre neurophénoménologique qui sous-tend le travail effectué au laboratoire de neuroscience éducationnelle de l'université Simon Fraser (ENGRAMMETRON). Ensuite, à titre d'illustration sont présentées des données psychophysiologiques, provenant d'études de cas pilotes en didactique de la géométrie, impliquant notamment l'électroencéphalographie (EEG) et l'électrooculographie (EOG). Trois techniques d'analyse des données brutes provenant de l'EEG sont abordées, soit l'analyse des sources, l'analyse spectrale et l'analyse statistique. Ces études de cas pilotes ont permis d'analyser le comportement électro-physiologique du cerveau et du corps, et de constater que ceux-ci s'activent différemment en fonction de la perception et du raisonnement face à la présentation de figures géométriques. Enfin, en conclusion, la pertinence de ce travail pour la théorisation en sciences de l'éducation, l'apprentissage et l'enseignement des mathématiques est soulignée et des pistes pour des études ultérieures en neuroscience éducationnelle sont suggérées. Ultimement, les auteurs espèrent faire valoir que la recherche en neuroscience éducationnelle, notamment celle s'appuyant sur l'inscription corporelle radicale de la pensée (Thompson et Varela, 2001), a sa place en sciences de l'éducation au XXlème siècle et peut s'avérer d'un apport fécond, tant au plan théorique que méthodologique.
\end{abstract}




\section{Introduction}

On est frappé de voir un physicien qui a libéré sa propre science des canons classiques du mécanisme et de l'objectivisme, reprendre sans hésitation, dès qu'il passe au problème philosophique de la réalité ultime du monde physique, la distinction cartésienne des qualités premières et des qualités secondes, comme si la critique des postulats mécanistes à l'intérieur du monde physique ne changeait rien à notre manière de concevoir son action sur notre corps, comme si elle cessait de valoir à la frontière de notre corps et n'appelait pas une révision de notre psychophysiologie (Maurice Merleau-Ponty, 2010, p. 1660).

On pourrait généraliser à bien des agents œuvrant dans le merveilleux monde de l'éducation ce remarquable passage du dernier ouvrage inachevé produit en 1962 par Merleau-Ponty. L'objet de cet article consiste à présenter des arguments en faveur de la recherche en neuroscience éducationnelle qui peut être bien davantage qu'un simple domaine d'application des neurosciences cognitive et sociale (Campbell, 2011). Ainsi, depuis deux décennies, les nouvelles connaissances décrivant les mécanismes physiologiques du cerveau bousculent nos conceptions de l'apprentissage (Sousa, 2011). Stimulées par ces nombreuses découvertes, les neurosciences cognitive (Byrnes, 2001) et sociale (Decety, 2011; ImmordinoYang, 2011) s'activent, entre autres, à trouver des applications potentielles en éducation. Par exemple les recherches sur le développement du cortex préfrontal ont produit des indications pour l'élaboration de stratégies d'apprentissage qui supportent et étayent les fonctions exécutives des adolescents (Lenroot et Giedd, 2006; Steinberg, 2007). En effet, certaines aires du cerveau associées au renforcement, à la motivation et à l'impulsivité se développent plus rapidement que les aires du cortex préfrontal qui pensent logiquement, qui soupèsent le pour et le contre et qui sont associées à l'autocontrôle. Ainsi, ces recherches ont permis de démontrer, qu'en plus d'interventions ciblant le développement cognitif des fonctions exécutives à l'adolescence, un autre type d'intervention, centré sur le développement d'une plus grande conscience et d'une plus grande autorégulation des émotions pouvait être bénéfique (Van Leijenhorst et al., 2010).

Par ailleurs, même si l'approche des neurosciences cognitive et sociale s'avère tout à fait légitime, des chercheurs en éducation se sentant interpellés directement ont commencé à intégrer les outils des neurosciences dans leurs propres recherches (Campbell, 2010; Lee et al., 2007). Une telle intégration ne fait toutefois pas l'unanimité. En effet, dans le monde des sciences de l'éducation, un scepticisme, sinon un déni, a dominé jusqu'à 
maintenant concernant l'utilité et la valeur des neurosciences pour mieux comprendre la cognition et l'apprentissage (Bruer, 1997; Churchland, 1980). Turner (2011), pour sa part, critique la relation entre neurosciences et éducation en arguant que présentement, il s'agit davantage d'un échange à sens unique, dominé par les neurosciences cognitives. Ainsi, malgré plusieurs initiatives de la recherche en neurosciences éducationnelles (voir Patten et Campbell, 2011), un scepticisme et une grande prudence prévalent encore (Snook, 2012). Pourtant, appréhender le cerveau comme notre organe de pensée et l'activité neuronale comme le reflet des processus de pensée conduit à mettre en lumière la nature de la pensée et ainsi de l'apprentissage. Les études du cerveau et de l'activité neuronale apparaissent donc d'intérêt fondamental pour les éducateurs et les enseignants.

La recherche en neuroscience éducationnelle, notamment celle ancrée dans la perspective neurophénoménologique de l'inscription corporelle de la cognition et de l'apprentissage, conçoit le cerveau comme l'organe de la pensée et de l'action (Campbell, 2011; Campbell et Dawson, 1995; MerleauPonty, 1962; Varela, Thompson et Rosch, 1991). Cette perspective a comme objet d'analyse l'être vivant, sentant, agissant, conscient et apprenant (Thompson, 2007); être qui se trouve situé et canalisé en fonction des opportunités et des contraintes émanant de son contexte historico-culturel (Pagé, Strayer et Reid, 2001; Vygotski, 1978). La neuroscience éducationnelle a ainsi le potentiel de devenir une entreprise transdisciplinaire qui embrasse radicalement les phénoménologies qui se trouvent corporellement inscrites, notamment dans le cerveau. Alors, bien que son domaine de recherche soit en partie informé par les théories, les méthodes et les résultats émanant des neurosciences, la recherche en neuroscience éducationnelle, située dans cette perspective neurophénoménologique, n'est pas limitée par eux, puisque son ancrage se trouve dans la riche tradition de la théorisation et de la recherche en sciences de l'éducation.

La recherche neuroscientifique en éducation regroupe donc à la fois des agents qui se définissent davantage comme des chercheurs en neurosciences cognitive ou sociale, appliquant leurs résultats à des problèmes éducationnels; ou comme des chercheurs en sciences de l'éducation, utilisant les méthodes et techniques des neurosciences cognitive et sociale. Peu importe leurs orientations ces chercheurs peuvent néanmoins adhérer aux mêmes paradigmes de recherche c'est-à-dire ceux associés à l'utilisation de l'imagerie cérébrale et à d'autres mesures psychophysiologiques même si fondamentalement ils sont animés par des vues philosophiques très différentes. À cet effet, il a été maintes fois souligné que les chercheurs devraient révéler leur posture épistémologique (Campbell, 
2011; Deschenaux et Laflamme, 2007; Kuhn, 1962), puisque celle-ci influence les méthodologies utilisées pour répondre aux questions de recherche et colore leurs interprétations des résultats obtenus.

L'objectif général de cet article s'arrime d'abord autour de la présentation des éléments du cadre neurophénoménologique qui sous-tend le travail effectué au laboratoire de neuroscience éducationnelle de l'université Simon Fraser (ENGRAMMETRON). À notre connaissance, celui-ci est le seul laboratoire de ce type hébergé dans une Faculté des sciences de l'éducation au Canada. Ensuite, quelques-unes des méthodes, des techniques et des types d'analyses utilisés dans ces recherches seront illustrés en présentant des données d'études de cas pilotes en didactique de la géométrie. II s'agit d'illustrer par ces résultats préliminaires comment la recherche en sciences de l'éducation peut être enrichie par l'apport de données psychophysiologiques. Enfin, en conclusion, la pertinence de ce travail notamment pour la théorisation en sciences de l'éducation, l'apprentissage et l'enseignement des mathématiques sera soulignée et des pistes pour des études ultérieures en neuroscience éducationnelle seront suggérées. Ultimement, les auteurs espèrent faire valoir que la recherche en neuroscience éducationnelle, notamment celle s'appuyant sur l'inscription corporelle radicale de la pensée (Thompson et Varela, 2001), a sa place en sciences de l'éducation au XXlième siècle et peut s'avérer d'un apport fécond, tant au plan théorique que méthodologique.

\section{Cadre théorique}

Depuis le dualisme Cartésien (Descartes, 1996) et d'autres avant lui, la nature des liens entre l'esprit (res cogitans) et le corps (res extensa) constitue un problème difficile, pour certains «le " problème difficile (Chalmers, 1998). Les tentatives matérialistes ou idéalistes pour réduire l'un à l'autre n'ont pas réussie à résoudre ce problème récurrent (Campbell, 2011) et les différentes positions philosophiques sont encore bien vivantes en sciences de l'éducation. II n'est donc pas question pour la recherche en neuroscience éducationnelle d'évacuer les différences épistémologiques importantes auxquelles s'abreuvent les multiples acteurs de la recherche en sciences de l'éducation. Ainsi, concernant le problème philosophique du lien entre l'esprit et le corps, notamment le cerveau, il n'est pas nécessaire, ni même souhaitable, que les chercheurs en neuroscience éducationnelle de diverses inclinaisons épistémologiques se trouvent à promouvoir des vues philosophiques identiques relatives à ces questions. Ceci étant dit, cela ne soustrait par le chercheur en neuroscience éducationnelle, comme dans 
d'autres domaines du savoir, de révéler la posture épistémologique et métathéorique qui sous-tend son travail (Winegar, 1996).

Le cadre épistémologique adopté par les auteurs de l'article concorde avec les positions de Merleau-Ponty (1962), et de Varela et al. (1991), qui soutiennent que la cognition est incarnée, c'est-à-dire inscrite corporellement, ce qui écarte totalement l'idée que les relations entre l'esprit, le corps et les comportements sont inaccessibles à l'étude scientifique. $\mathrm{Si}$, contrairement aux croyances traditionnelles postulant que l'esprit est séparé de la matière (Descartes, 1996), le corps, notamment le cerveau, et l'esprit sont perçus comme formant un tout indissociable, il doit nécessairement y avoir une façon d'observer physiquement les processus cognitifs et d'apprentissage et donc de les étudier (Campbell, 2010). Ainsi, l'esprit, le corps et le cerveau peuvent être appréhendés comme des aspects de la même unité d'analyse " espritcorps-cerveau ", qui peut être étudiée et devenir un objet d'étude de la neuroscience éducationnelle. Cette entité esprit-corps-cerveau, conçue comme étant ontologiquement unifiée, est semblable à la notion de Chair dans le Visible et l'Invisible (voir Merleau-Ponty, 2010). Ainsi, le cerveau peut-être vu comme le point de convergence de la conscience dans le corps, ce qui par ailleurs, ne présume pas que l'esprit-cerveau est limité par la frontière de la chair (Campbell, 2011).

Au plan philosophique, le cadre de l'inscription corporelle radicale de l'esprit (Thompson et Varela, 2001) offre par ailleurs une voie du milieu entre les formulations monistes, proposant que tout est esprit (idéalistes), ou que tout est matériel (matérialistes); et les postions dualistes extrêmes, soutenant une séparation hermétique entre l'esprit et le corps (Campbell et Dawson, 1995; Handscomb, 2007). De cette façon, dans un sens matérialiste, bien qu'en tant qu'êtres physiques, nous existions dans le monde, dans un sens constructiviste et phénoménologique, nous sommes également conscients du monde qui existe aussi en nous (Campbell, 2011).

L'inscription corporelle, telle que définie par Varela et al. (1991) inclut à la fois la vision du corps, comme une structure vivante expérientielle; et le corps comme le milieu ou le contexte d'expression de l'esprit. L'inscription corporelle radicale (Thompson et Varela, 2001), présuppose donc que n'importe quel changement dans l'expérience subjective doit, en principe, se manifester objectivement, d'une certaine manière, comme un changement dans le cerveau, le corps et les comportements, et vice-versa. Ce point de vue de l'inscription corporelle radicale de l'esprit, circonscrit à l'unité d'analyse esprit-corps-cerveau, conduit à chercher des liens entre l'expérience subjective et le comportement corporellement inscrit. 
Au plan méthodologique, cette perspective commande de croiser des données provenant de méthodes dites à la première et à la troisième personne (Varela et Shear, 1999). Afin de mieux comprendre les changements dans l'expérience subjective (à la première personne) des apprenants, il s'agit notamment d'observer et de mesurer les changements dans leurs comportements neuronaux (à la troisième personne), donc dans leur cerveau, en utilisant pour ce faire diverses méthodes comme l'électroencéphalographie (EEG). Ces données psychophysiologiques peuvent ensuite être reliées à leurs expériences subjectives. Ainsi, Lutz, Lachaux, Martinerie et Varela (2002) ont démontré que le discours phénoménologique des sujets, en lien avec la présentation d'illusions tridimensionnelles, peut être utilisé pour détecter et interpréter les processus neuronaux. En somme, la cognition incarnée implique que tout changement dans l'expérience subjective doit, d'une certaine façon, se manifester d'une manière objective par des changements physiologiques et comportementaux.

La recherche en neuroscience éducationnelle doit s'activer à établir des ponts, ou à tout le moins, de réduire la distance entre les diverses théories et les multiples méthodes consacrées aux processus associés à l'enseignement et à l'apprentissage. Dans la mesure où les comportements cognitifs et l'apprentissage deviennent directement observables, notamment suite aux avancées émergeant des découvertes en neurosciences, il devient impérieux pour la recherche en sciences de l'éducation d'intégrer des mesures psychophysiologiques. Ceci permettra de compléter les diverses données issues d'entrevues, d'auto-évaluations, d'évaluations formelles des apprentissages, de notes d'observation participante ou d'observation systématique des comportements, etc. Des analyses croisées de ces différentes sources de mesure permettront de jeter un nouveau regard sur les processus d'apprentissage afin d'ultimement mieux informer et former les éducateurs et les enseignants. C'est en ce sens que la recherche en neuroscience éducationnelle s'avère une avenue prometteuse pour les sciences de l'éducation.

\section{Méthodes et techniques de la recherche en neuroscience éducationnelle chez ENGRAMMETRON}

Les études en neuroscience éducationnelle qui sont réalisées par les membres du laboratoire ENGRAMMETRON font appel à des méthodes observationnelles propres à la recherche en éducation, notamment, les autoévaluations, les évaluations des apprentissages, des motivations, des représentations subjectives et autres éléments d'intérêt. Parmi ces méthodes se comptent l'utilisation de différents tests psychométriques valides, des 
enregistrements audiovisuels des comportements, comprenant la capture d'écran et des touches d'un ordinateur par des apprenants. En plus du recours à ces méthodes classiques, les études d'ENGRAMMETRON innovent en recueillant des données issues d'une variété de méthodes et techniques psychophysiologiques, comme par exemple: 1) l'électroencéphalographie (EEG), qui mesure les ondes cérébrales; 2) l'électrocardiographie (EKG), qui mesure l'activité cardiaque; 3) l'électrooculographie (EOG), qui repose sur les variations du potentiel de repos cornéo-rétinien, lors des déplacements du globe oculaire; 4) l'oculométrie (eye-tracking), qui comprend un ensemble de techniques permettant de suivre le mouvement de l'œil (sur un écran d'ordinateur), dans le but de savoir ce qu'une personne regarde; et 5) des mesures physiologiques diverses (respiration, température, etc.).

Le principal défi technologique pour l'équipe d'ENGRAMMETRON est d'intégrer, à l'aide du logiciel Noldus Observer XT, tous ces différents types de données de manière synchronisée dans le temps, puisque chacun a son propre cadre temporel. À titre d'exemple, la Figure 1 présente une capture d'écran d'une étude pilote pour laquelle l'ensemble des sources d'information est synchronisé dans le temps : l'électroencéphalogramme des électrodes situés sur le lobe frontal (Canal B1) se trouve en P1; se trouvent ensuite dans l'ordre: le taux de battements du cœur par minute en P2; les battements du cœur provenant de l'électrocardiogramme (EKG) en P3; l'électrooculographie (EOG) occupe 4 canaux (2 électrodes pour les mouvements horizontaux en P4 et P7 et 2 pour les verticaux en P5 et P6); les mouvements musculaires (EMG) mesurant la tension dans le bas du cou en P8, la respiration en P9; un canal pour le signal temporel en P10, et finalement, l'enregistrement vocal en P11.

Les figures géométriques présentées au participant se retrouvent en V1 et sont accompagnées d'une capture d'écran du pistage de l'œil; les enregistrements vidéoscopiques du sujet sont présentés en V2 et V3 et l'ensemble des données brutes de l'EEG en V4. Toutes ces données, associées au même moment dans le temps, permettent une validation croisée qui s'avère un atout indéniable, notamment pour identifier les artefacts potentiels associés à l'EEG. Dans cet article, à titre d'exemples, nous nous limiterons à présenter des résultats préliminaires issus d'études de cas impliquant l'électroencéphalographie (EEG) et l'électro-oculographie (EOG). 


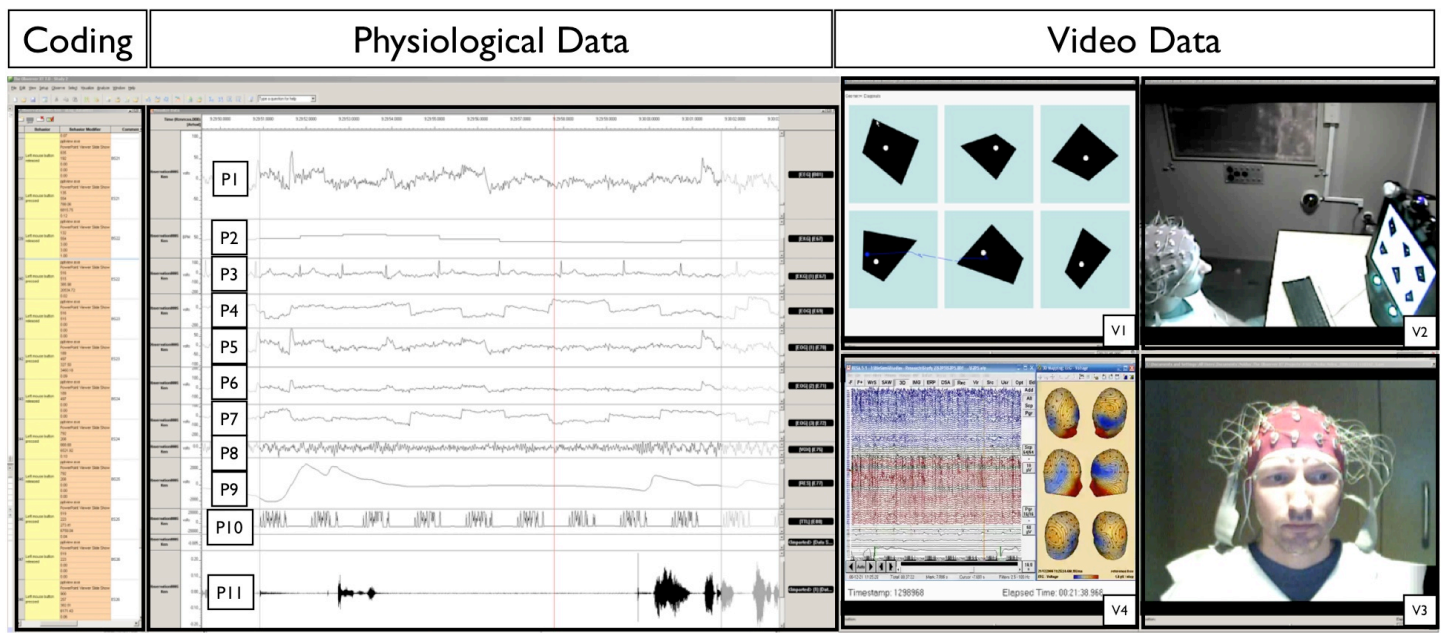

Figure 1. Capture d'écran des données comportementales et physiologiques à l'aide du logiciel Noldus Observer XT

\subsection{L'électroencéphalographie (EEG)}

Les données provenant de l'électroencéphalographie mesurent indirectement le champ électromagnétique généré à l'intérieur du cerveau, c'est-à-dire les changements rapides de tension potentielle sur le cuir chevelu. L'activité électrique enregistrée sur le cuir chevelu est le résultat de l'activité de populations de neurones (Harmon-Jones et Peterson, 2009). En effet, parce que l'activité générée par un neurone est petite, l'activité enregistrée sur le cuir chevelu est la synchronie de l'activité intégrée d'un ensemble de neurones. L'électroencéphalographie est donc une méthode pour déterminer l'activité électrique générée par le cerveau, en mesurant les changements spatiotemporels dans le potentiel électrique à la surface du cuir chevelu. L'EEG est possible à cause des mouvements subtils d'électrons libres identifiables sur le cuir chevelu, en réponse au champ bio-électromagnétique généré par l'activité neuronale dans le cerveau. La Figure 1 illustre en V4 cette activité du cerveau pensant. La vidéo a été ralentie à $1 / 50$ ième par rapport au temps réel. En effet, la vitesse de la pensée est très rapide. II existe plusieurs approches liées à l'analyse de l'EEG. Au laboratoire ENGRAMMETRON, les données brutes provenant de l'EEG sont analysées en trois étapes : l'analyse des sources, l'analyse spectrale, et l'analyse statistique. 


\subsection{Analyses des données de l'EEG}

\subsubsection{Analyse des sources d'énergie}

L'EEG enregistre l'évolution du potentiel de tension électrique sur le cuir chevelu qui est causée par l'activité neuronale dans le cerveau. Le signal EEG brut (Figure 1, V4) est une forme d'onde complexe qui peut être analysée dans le domaine temporel ou fréquentiel. Le traitement de l'aspect temporel se fait typiquement avec des devis et analyses impliquant le potentiel relatif (Bartholow et Amodio, 2009). Cet article s'attarde plutôt aux analyses fréquentielles de l'EEG, où la fréquence s'exprime en hertz $(\mathrm{Hz})$ ou cycles par seconde.

L'analyse des sources permet de séparer et de localiser les sources d'énergie réparties dans différentes parties du cerveau. Pour ce faire, les données enregistrées sur le cuir chevelu à l'aide de dipôles électriques sont analysées dans le but de générer des modèles quant aux différentes régions du cerveau activées. II existe plusieurs types d'équipement ayant recours à un nombre différent d'électrodes (64, 128 ou 256 canaux). Chacun présente des avantages et des désavantages (voir Michel et al., 2004; Vaisanen, 2008), en lien avec la précision de la résolution spatiale qui augmente avec le nombre de canaux. Au laboratoire ENGRAMMETRON, même si ce n'est pas optimal, 64 canaux sont jugés comme offrant néanmoins une résolution spatiale suffisante pour le type de source régionale utilisé. La Figure 1, en V4, illustre les 64 canaux de données EEG enregistrées sur le cuir chevelu. Les 32 premiers canaux proviennent de l'énergie électrique générée par l'hémisphère gauche, alors que la deuxième série de 32 canaux provient de l'hémisphère droit.

Le problème inverse posé par l'analyse des sources consiste à transformer des données acquises à partir de capteurs localisés à l'extérieur du crâne, en sources électromagnétiques localisées à l'intérieur du cerveau. Résoudre ce problème requiert l'utilisation de modèles mathématiques gouvernant la manière dont les signaux sont présumés être distribués et propagés à travers différentes couches, telles le cerveau, le crane et le cuir chevelu (Grech et al., 2008). L'analyse des sources est donc une forme de modèle inverse (Campbell, 2004) qui implique de déterminer les régions sous-jacentes à l'activité du cerveau qui sont associées aux mesures prises sur le cuir chevelu pendant une certaine période déterminée. Une méthode multiple de dipôle de courant équivalent discret (multiple discrete equivalent current dipoles) est utilisée, afin d'identifier les sources provenant des régions du cerveau (Scherg, 1990). Comme le démontre la Figure 2, à gauche, les 64 canaux de données enregistrées sur le cuir chevelu sont ainsi réduits à 15 
différentes sources d'activité, réparties sur l'ensemble du cerveau et associées à l'activité totale de ces 15 régions spécifiques du cerveau.

\subsubsection{Analyse spectrale}

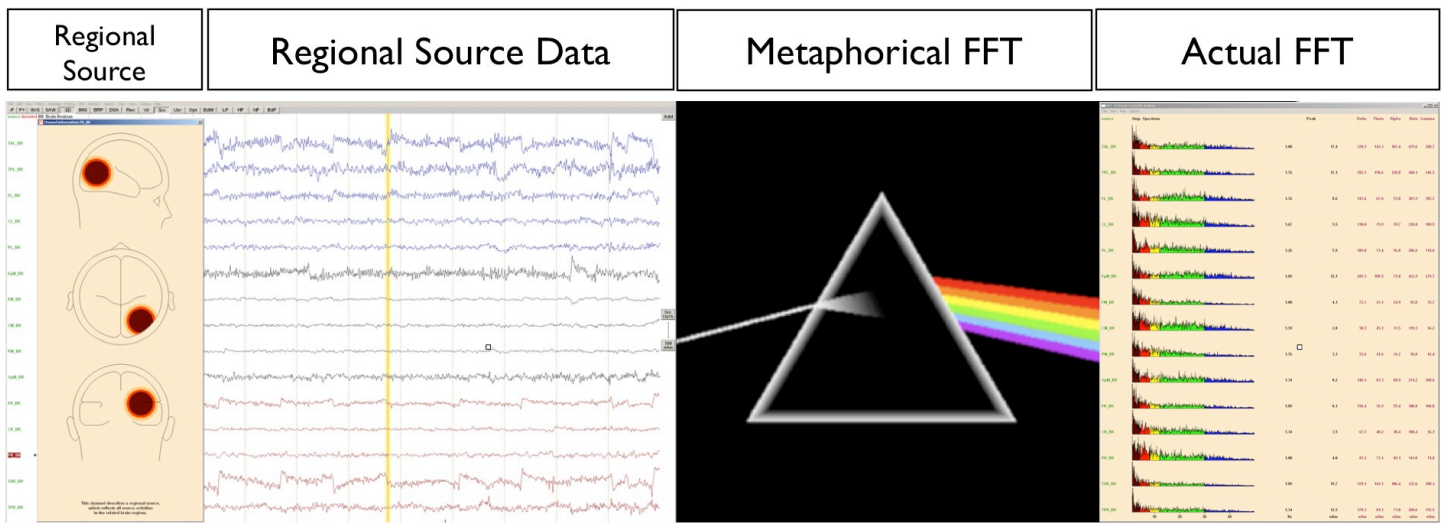

Figure 2. L'analyse spectrale par la Transformée de Fourier Rapide (FFT)

Tel qu'illustré métaphoriquement dans la Figure 2, de la même façon que l'analyse spectrale des étoiles nous a permis une meilleure compréhension de notre univers, l'analyse spectrale des ondes cérébrales permet de mieux comprendre l'activité du cerveau. La Transformée de Fourier Rapide (FFT dans la Figure 2) est une application du traitement des signaux se trouvant à être l'équivalent mathématique d'un prisme. L'utilisation de la Transformée de Fourier permet de décomposer la source de données régionales en 5 gammes de fréquence (appelée delta de 0.5 à $4 \mathrm{~Hz}$, thêta de 4 à $7.5 \mathrm{~Hz}$, alpha de 7.5 à $14 \mathrm{~Hz}$ (hertz), bêta de 14 à $30 \mathrm{~Hz}$ et gamma, de $30 \mathrm{~Hz}$ et plus) qui sont bien connues pour être associées à différents aspects de la fonction cognitive (Figure 2). Par exemple, les ondes Beta sont associées à un niveau élevé de conscience éveillée, au raisonnement critique et logique. La Figure 2 représente le processus d'analyse à partir des données brutes enregistrées sur le cuir chevelu, jusqu'à l'analyse spectrale de sources régionales dans le cerveau.

\subsubsection{Analyses statistiques}

Dans la foulée des deux premières analyses (des sources et spectrale), les valeurs de puissance équivalentes à 75 variables dépendantes (15 sources $x$ 5 gammes de fréquences $=75$ ) sont associées à divers aspects du comportement qui peuvent désormais être analysés à l'aide d'outils 
statistiques usuels en sciences de l'éducation et en psychologie, tels que l'ANOVA et la MANOVA.

\section{4. Études pilotes}

Afin d'illustrer les méthodes et techniques qui ont brièvement été exposées, les résultats préliminaires de deux études pilotes en neuroscience éducationnelle des mathématiques, qui s'intéresse à la perception d'images et au raisonnement en géométrie sont présentés (Campbell et al., 2009). En accord avec le modèle théorique de l'inscription corporelle radicale de la cognition et de l'apprentissage (Campbell, 2001, 2003; Varela et al., 1991; Thompson et Varela, 2001), les manifestations physiologiques de la perception de figures géométriques et du raisonnement mathématique sont identifiées par l'activité du cerveau et du corps, ce qui peut ensuite être corrélé avec des observations du comportement et des auto-évaluations du sujet.

La démarche vise à identifier quels aspects des figures géométriques présentées à un apprenant sont perçus subjectivement d'instant en instant et comment l'esprit-corps-cerveau y réagit. Cela présuppose qu'en plus des verbalisations, les ondes cérébrales de l'apprenant et le point focal de ses yeux sur les figures géométriques présentées soient enregistrés. Ces recherches visent ultimement à mieux situer les diverses modalités de la cognition géométrique, afin de mieux comprendre l'apprentissage, l'enseignement et l'évaluation des mathématiques.

\subsection{La perception géométrique}

La première étude de cas se centre sur la manifestation physiologique du changement de perception quand un sujet est confronté à la présentation de figures géométriques. Même si la psychologie et la philosophie s'intéressent depuis longtemps à la perception (Merleau-Ponty, 1962), les réseaux neuronaux sous-jacents à ce phénomène complexe sont encore mal compris (Pitts, Martinez, Stalmaster, Nerger et Hillyard, 2009). L'objectif ici est de simplement démontrer comment les changements dans la présentation de stimuli se manifestent par des changements dans les ondes cérébrales, telles qu'enregistrées par l'électroencéphalographie.

Dans ce projet pilote, le Cube de Necker a été utilisé. Tel qu'illustré en bas à gauche de la Figure 3, cette figure ambiguë dessinée par Necker en 1832, présente les faces avant et arrière du cube, d'égales mesures, contrairement au tracé traditionnel dans lequel la face avant est plus grande que la face 
arrière. II s'ensuit que deux images du cube apparaissent et le cerveau passe d'une représentation à l'autre. De tels types de figures ambiguës s'avèrent utiles pour analyser les bases neurologiques de l'expérience sensorielle, puisque les «inputs sensoriels» demeurent fixes, tandis que se produisent dans le cerveau des changements de perception associés à des changements de représentation (Pitts et al., 2009: Shimaoka, Kitajo, Kaneko et Yamaguchi. 2010). Cette démarche vise ainsi à évaluer si un participant est en mesure de percevoir le cube avec la face vers le haut ou s'il perçoit le cube avec la face vers le bas.

Plus précisément, l'étude pilote vise ici à répondre aux questions suivantes : Est-ce que des changements dans l'état cérébral peuvent être détectés par l'EEG en lien avec les changements de stimuli? Est-ce que la complexité de l'activité cérébrale s'accroit avec la présentation de stimuli géométriques plus complexes?

Une fois le participant relié aux différents instruments, une mesure EEG de base, soit au repos, est enregistrée. Trois stimuli illustrés dans le bas de la Figure 3 ont ensuite été présentés au participant dans l'ordre suivant : Un écran blanc pendant 5 secondes; suivi d'un carré pendant 5 secondes; et enfin, le cube de Necker qui reste sur l'écran jusqu'à ce que le participant indique que sa perception du cube a changé. II indique ce changement, soit en appuyant sur la touche indiquant une flèche vers le haut, pour un mouvement du bas vers le haut; soit en appuyant sur la touche indiquant une flèche vers le bas, pour un mouvement du haut vers le bas. Les deux premiers stimuli servent de mesure de contrôle de l'état de base visuel et neuronal (Gaetz, Weinberg, Rzempoluck, et Jatzen, 1989). Cette présentation des trois stimuli est répétée pour un total de vingt cycles, après quoi un autre moment de mesure de base au repos est enregistré. 


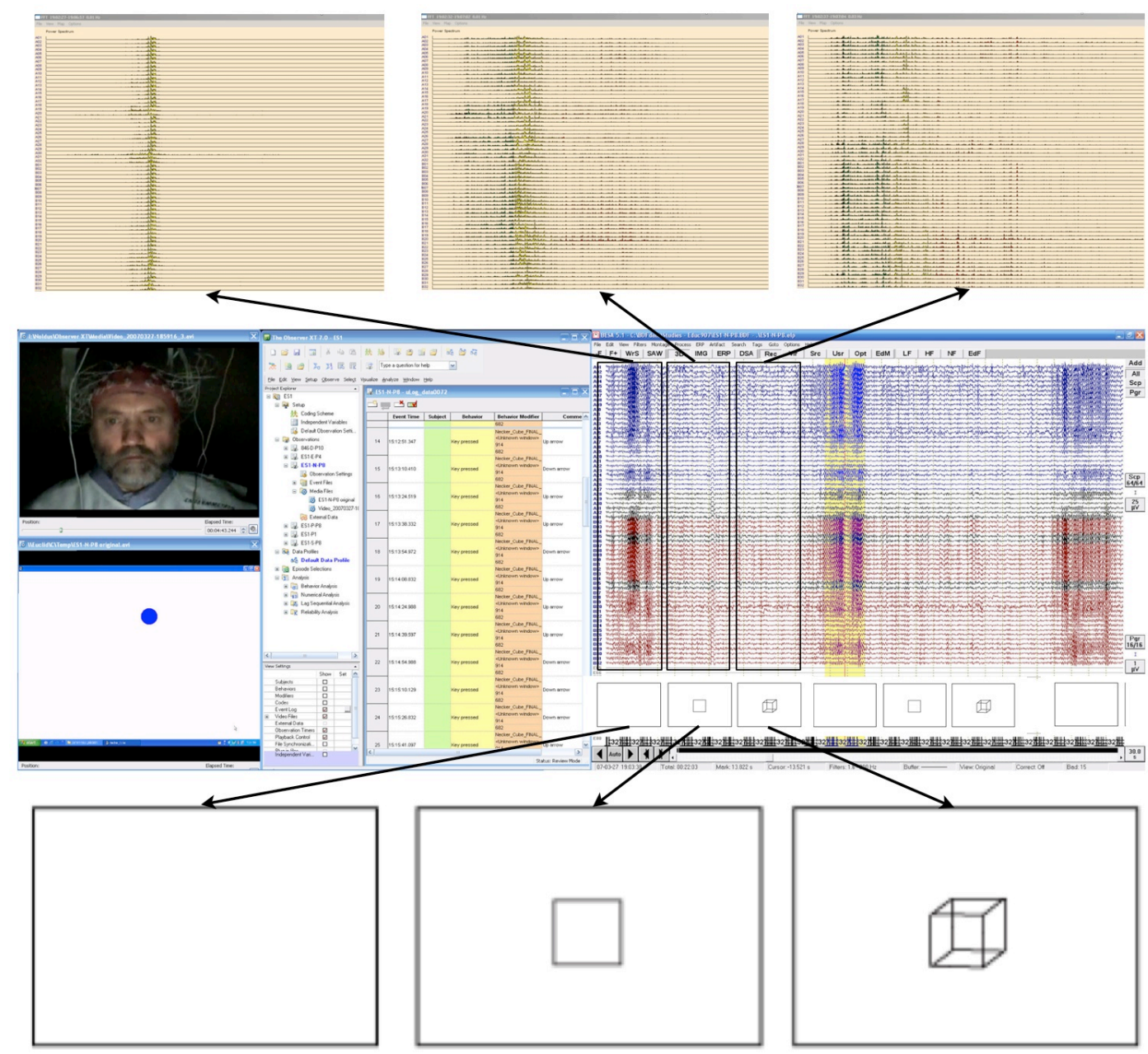

Figure 3. La perception géométrique. En bas, les trois stimuli présentés au sujet (un écran blanc, un carré et le cube de Necker); au milieu, la capture des informations et les données des 64 électrodes de l'EEG; en haut, les caractéristiques spectrales associées à chaque stimulus.

À titre d'exemple, au milieu de la Figure 3, est présenté un EEG des données associées à chaque stimulus. Sur la partie gauche, le participant regarde un fond d'écran blanc. Le point situé sur la partie gauche indique la position de son regard sur l'écran de l'ordinateur. Dans le centre de l'image, sont représentés les panneaux avec clé de codage AVC (Advanced Video Coding), pour la capture des informations; et les données des 64 électrodes de l'EEG. Sans s'attarder à l'analyse des sources, la Figure 3 permet de constater que des différences sont discernables dans les caractéristiques spectrales de chacun de ces stimuli, c'est-à-dire dans le type d'« arc-en-ciel » des ondes cérébrales enregistrées sur le cuir chevelu du participant. Celles-ci 
sont associées respectivement au moment où il observe le fond d'écran blanc, celui du carré et du cube, dans cet ordre.

Toujours dans la Figure 3, en haut et à gauche, l'indice numérique représente la moyenne pour les 20 cycles de présentation de la première seconde de I'EEG face à l'écran blanc. Pour chaque exposition est appliquée la Transformée de Fourier Rapide. Ce processus est ensuite répété et les données cumulatives sont utilisées pour construire la moyenne de la première seconde de l'exposition à la figure du carré, auquel est appliquée la Transformée de Fourier Rapide (Figure 3, au milieu). Et maintenant, une fois de plus, l'ensemble du processus est répété pour le cube (Figure 3, à droite). De prime abord, à partir des données de l'EEG obtenues, il n'est pas facile de discerner une grande différence entre l'exposition au carré et l'exposition au cube.

II faut cependant noter ici que l'amplitude la plus élevée est associée à l'étape où le participant est face à un écran vide. La progression liée à chaque essai est ainsi caractérisée par une puissance accrue dans les ondes alpha (8$14 \mathrm{~Hz}$ ). Ces augmentations de puissance sont liées aux moments où le participant regardait l'écran blanc. Ces ondes alpha sont typiquement associées à une condition de relaxation avec les yeux fermés. Pourtant notre participant avait les yeux ouverts. Cette observation peut être mise en lien avec les résultats de Knyazev, Savastyanov et Levin (2005), qui rapportent aussi un niveau d'alerte consciente ou d'attention qui pourrait être activé par l'expectative de la tâche à venir. Bien sûr ces données préliminaires exigent une analyse plus poussée et une réplication avec d'autres sujets, mais pour l'instant, il est possible de discerner diverses caractéristiques spectrales de l'EEG du cuir chevelu, en les associant aux différents stimuli géométriques.

II existe deux problèmes qui doivent être traités. Tout d'abord le ratio signal/bruit est habituellement perçu comme ne pouvant pas produire des résultats fiables à partir d'un seul enregistrement. Afin de contourner ce problème, ENGRAMMETRON a été construit de façon à avoir un excellent ratio signal/bruit. Ensuite, il y a la plupart du temps d'autres éléments qui viennent colorer l'activité de l'esprit-corps-cerveau. Alors comment discerner une manifestation d'activité cognitive de d'autres activités ayant une magnitude équivalente ou même plus grande? Chez ENGRAMMETRON, l'analyse en composante indépendante (voir Makeig, Bell, Jung et Sejnowski, 1996) est utilisée afin de distinguer statistiquement les manifestations non reliées à la fonction cognitive. 


\subsection{Le raisonnement géométrique}

Avant de présenter la deuxième étude pilote, voici une description de l'utilisation de l'électro-oculographie qui permet notamment de mesurer des potentiels électriques continus cornéen-rétinien induits par la rotation des yeux grâce à des électrodes placées autour des yeux. La cornée de l'œil est chargée positivement par rapport à l'arrière de l'œil. Ce potentiel se manifeste comme s'il s'agissait d'un dipôle électromagnétique unique orienté de la rétine à la cornée (Malmivuo et Plonsey, 1995).

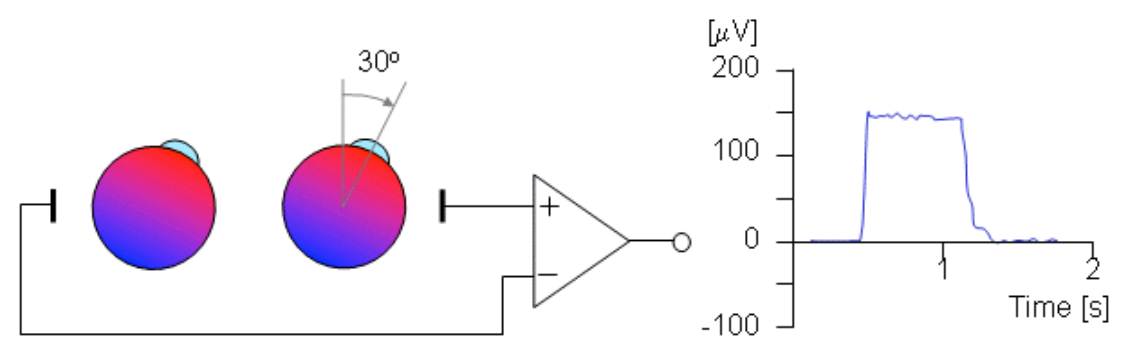

Eyes moving $30^{\circ}$ to the right
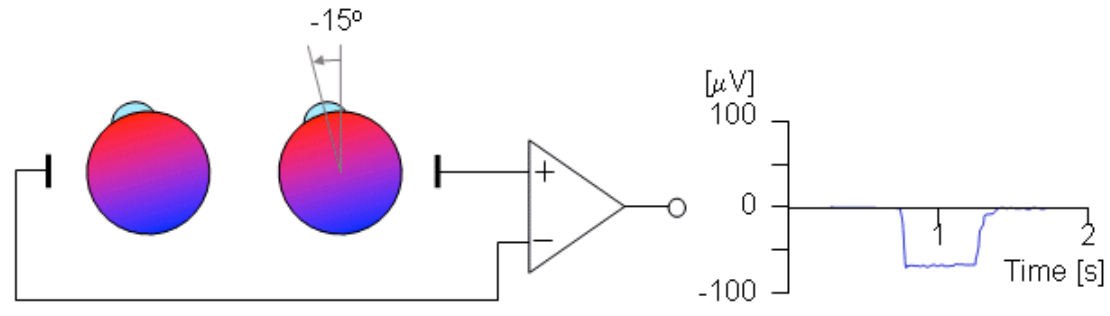

Eyes moving $15^{\circ}$ to the left

Figure 4. Technique de l'électro-oculographie (EOG) représentant les mouvements horizontaux des yeux. Illustration tirée de Malmivuo et Plonsey (1995).

En consultant la Figure 1 au début de l'article, on peut voir, en plus des transducteurs attachés au chapeau porté par le sujet pour l'électroencéphalographie, l'emplacement des capteurs EOG. Le point d'attention se situe aux canaux P4 à P7, qui correspondent aux signaux de l'EOG. On se rappellera que deux électrodes sont associées aux mouvements horizontaux en P4 et P7 et deux aux verticaux en P5 et P6. Sur la Figure 4, l'information vise à décrire comment le signal EOG est généré par le changement dans le potentiel cornéen-rétinien. Par exemple, sur l'illustration, les mouvements horizontaux des yeux sont présentés et la polarité du signal est positive relativement à l'électrode avec laquelle l'œil se meut. 
Au plan de la recherche en neuroscience éducationnelle, trois applications découlent des mesures de l'EOG. Tout d'abord, cette technique permet de mieux comprendre la fonction cognitive. Ainsi, des études ont utilisé le pistage de l'œil comme indicateur de processus attentionnels et mnémoniques (Kramer et McCarley, 2003). L'étude de la réponse pupillaire a permis d'associer les variations de ce comportement avec la charge cognitive associée à une tâche (Just, Carpenter et Miyake, 2003). Les recherches utilisant cette technique démontrent que les clignotements et les mouvements des yeux sont des indicateurs de fonctionnement cognitif. Par exemple, les taux de clignotements des sujets diminuent de façon significative face à des tâches visuellement exigeantes (Fogarty et Stern, 1989).

Une autre application des mesures EOG est de permettre d'atténuer les artefacts liés aux mouvements des yeux en électroencéphalographie. En effet, les mouvements de saccade des yeux produisent des artefacts qui s'avèrent plus difficiles à identifier. L'analyse en composantes indépendantes peut être utilisée, afin de bien séparer les différentes sources des données; et ainsi, grâce à l'EOG, à identifier les composantes qui sont dues aux mouvements saccadés des yeux. Enfin, une troisième application des données EOG pour la recherche en neuroscience éducationnelle est de permettre de calibrer l'activité interne du cerveau avec un comportement manifeste, afin de mieux situer et comprendre un phénomène d'apprentissage.

Ce qui nous mène à notre deuxième étude pilote. Si la perception implique que nous voyons un élément présent dans notre champ sensoriel, le raisonnement implique la réflexion, souvent en comparant une chose avec une autre pour essayer d'identifier les relations de différences ou de similarité qui existent entre-elles.

De la même façon que pour la première étude pilote, il s'agit de vérifier s'il est possible de discerner des différences dans l'EEG du participant, mais cette fois-ci en fonction des rondes d'exposition à des diapositives illustrant des figures géométriques. Par exemple, les mesures EEG associées à la première seconde d'exposition de la première ronde [E1B] peuvent être comparées à celles de la première seconde d'exposition aux mêmes diapositives lors de la troisième ronde [E3B]. Dans la première ronde, il s'agit de stimuli nouveaux, misant davantage sur la perception, alors qu'à la troisième reprise, les participants sont beaucoup plus familiers avec les stimuli. En conséquence, a été formulée l'hypothèse qu'à la troisième ronde, la cognition serait davantage orientée vers la reconnaissance de mots, puisque dans cette ronde, l'attention du participant est dirigée vers le coin gauche de chaque diapositive, où un terme mathématique reliant 
conceptuellement cinq des six schémas est présenté. La différence entre la première et la troisième ronde devrait être détectée par l'activité cérébrale du participant.

Dans cette étude, 45 diapositives sont utilisées (Figure 5, gracieuseté de Dehaene, Izard, Pica, et Spelke, 2006). Chaque diapositive comporte 6 schémas. Cinq de ces schémas sont liés par un seul concept géométrique. Le participant a pour tâche d'identifier le schéma qui n'est pas conforme aux autres. L'objectif est ici de simplement démontrer comment les ondes cérébrales du participant changent depuis la perception initiale des diagrammes jusqu'aux considérations subséquentes sur ces mêmes diagrammes une fois qu'il a eu l'opportunité de réfléchir sur ceux-ci.

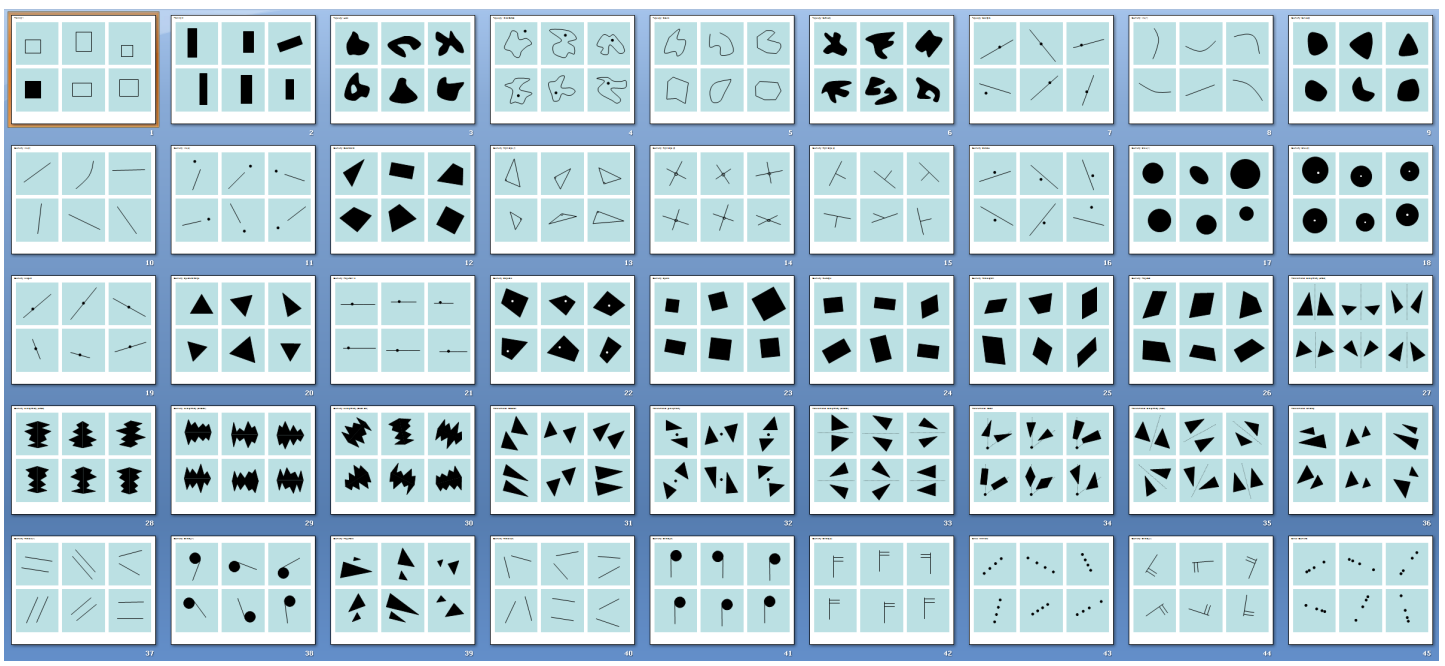

Figure 5. Stimuli géométriques de Dehaene, Izard, Pica et Spelke (2006).

Comme on peut le constater, dans de nombreuses diapositives, il est assez facile de déterminer le diagramme qui n'est pas relié aux autres, tandis que dans d'autres, la tâche est nettement plus subtile. Cependant, ce n'est pas vraiment ce qui nous intéresse ici. Dans cette étude pilote, toutes ces diapositives ont été présentées au participant à trois reprises. Une première fois, la tâche était simplement de déterminer le schéma qui n'est pas conforme à chaque diapositive, en cliquant sur ce diagramme. La deuxième fois, le participant, par la méthode du rappel stimulé (Tochon, 1996) a été invité à " parler à voix haute " au sujet de son expérience de la première fois. La troisième fois, son attention a été attirée sur le concept qui reliait 5 des 6 figures et qui a été présenté sur le côté supérieur gauche de chaque diapositive (Figure 6). Cette fois, on a demandé au participant, selon une 
méthode s'apparentant à l'entretien d'explicitation (Vermersch, 1994), de "penser à voix haute » sur la façon dont son expérience aurait pu être différente s'il avait eu cette notion dans son esprit avant de déterminer le schéma qui n'était pas en conformité avec les cinq autres. Par exemple, dans la Figure 6 représentant la diapositive 22, le terme qui est présenté au sujet dans le coin supérieur gauche et qui permet de relier conceptuellement 5 des 6 schémas est «diagonales ». Les données audiovisuelles du pistage de l'œil et les données physiologiques (EEG, EOG) sont ensuite intégrées et synchronisées pour l'analyse.

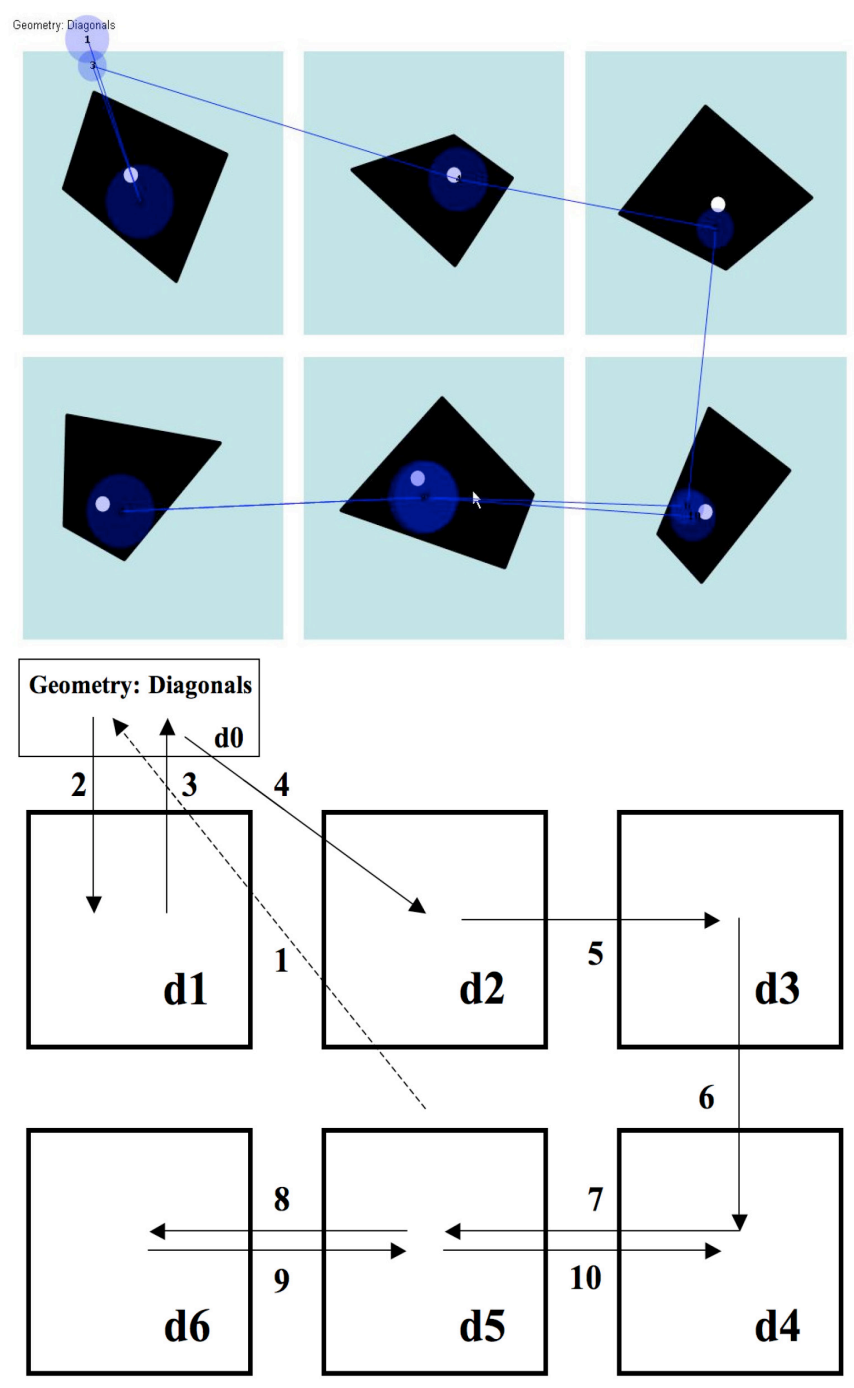

Figure 6. Diapositive 22. En haut, les mouvements des yeux sur l'écran durant la présentation de la diapositive 22 lors de la troisième ronde; en bas, la schématisation de ces mouvements. 
Afin de vérifier l'hypothèse de différences dans l'activité cérébrale du participant entre la première et la troisième ronde d'exposition aux 45 diapositives, une analyse des sources a donc été réalisée, puis, comme auparavant, une analyse spectrale. Enfin, 75 analyses ANOVA séparées $(\mathrm{N}=45$, puisque nous utilisons la première seconde de données pour chacune des trois rondes de présentation des 45 diapositives) permettent de déterminer pour quelles variables il est possible d'observer une puissance spectrale significativement plus grande (pour chacune des cinq gammes de fréquences EEG, et pour chacune des 15 différentes régions du cerveau) dans les ondes cérébrales du participant lors de la première présentation en comparaison avec la troisième.

L'objectif étant ici d'illustrer les différences reflétées par l'EEG dans la perception et le raisonnement face à des figures géométriques, seuls certains résultats préliminaires sont présentés. Dans le haut, et à gauche, de la Figure 7 , les lignes indiquent les différentes régions du cerveau. Celles du haut sont plus proches de l'avant de la tête alors que les plus basses sont associées à l'arrière. Les colonnes indiquent les gammes de fréquences (delta à gamma). Les valeurs $" p$ » des analyses ANOVA significatives $(p<.05)$ sont présentées en haut à gauche dans la Figure 6. Elles indiquent plus de puissance au début de l'épisode 1 (E1B) dans les gammes de fréquence élevées (gamma et bêta) dans la région frontale du cerveau, en comparaison avec le début de l'épisode 3 (E3B). 


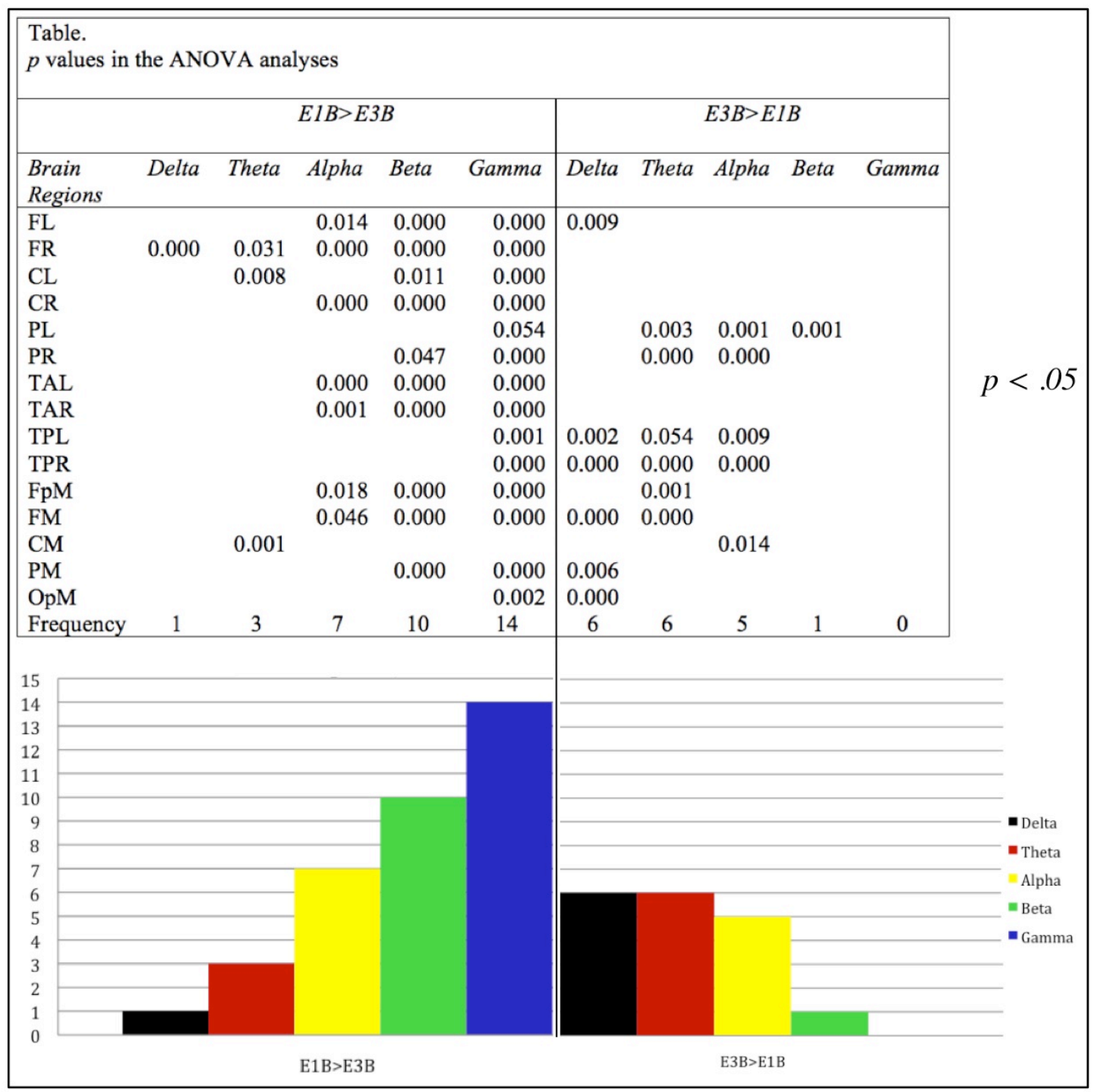

Figure 7. Résultats de l'EEG. E1B représente la première seconde de l'EEG de la première ronde d'exposition à chacune des 45 diapositives; E3B représente la première seconde de l'EEG de la troisième ronde d'exposition à chacune des 45 diapositives. Le bas de la figure illustre par un histogramme les fréquences des résultats significatifs de la partie supérieure.

Sans surprise (Figure 7, en haut, à droite), c'est l'inverse qui est statistiquement vrai, lorsque l'on compare le pouvoir au début de l'épisode 3 par rapport au début de l'épisode 1 . Les valeurs $p(p<.05)$ indiquent une plus grande puissance dans les basses gammes de fréquence, vers l'arrière de la tête. 
L'illustration au bas de la Figure 7 reprend, sous un autre angle, les mêmes résultats. Ils indiquent la fréquence des différences statistiquement détectables dans la puissance des bandes de fréquence au regard des régions du cerveau. En bas à gauche se trouve la fréquence des différences de l'épisode 1, qui est plus élevée que celle de l'épisode 3 . En bas à droite se trouve la fréquence de l'épisode 3 , qui est plus élevée que celle de l'épisode 1. Dans cette illustration, les gammes de fréquences vont du bas vers le haut. Durant la première seconde d'exposition aux 45 diapositives de l'épisode trois, les ondes cérébrales du participant démontrent une plus grande puissance dans les hautes fréquences gamma $(30-50 \mathrm{~Hz})$ et beta $(14-30 \mathrm{~Hz})$, et moins dans les basses fréquences thêta $(4-7,5 \mathrm{~Hz})$ et delta $(0-4 \mathrm{~Hz})$ durant la première seconde d'exposition aux 45 diapositives de l'épisode 1. Par ailleurs, les ondes alpha $(8-14 \mathrm{~Hz})$ révèlent plus de puissance dans les régions frontales du cerveau dans l'épisode 1 et encore davantage dans les régions postérieures dans l'épisode 3 .

Afin d'expliquer ces résultats préliminaires, l'hypothèse formulée suggère que lors de la première présentation des diapositives, le participant se trouvait dans un état d'esprit plus ciblé et plus attentif. Alors qu'à la troisième présentation il était beaucoup plus familier avec les diapositives et donc il a potentiellement davantage fait appel à sa mémoire à ce moment-là. En outre, son attention pour la troisième passation était également plus orientée vers des préoccupations linguistiques puisqu'il essayait de lire la partie supérieure gauche des diapositives et d'en comprendre le sens (Figure 6). D'ailleurs, en lien avec ces observations, des études (Bastiaansen, Berkum et Hagoort, 2002) ont démontré que la mémoire, la lecture et la compréhension ont été mises en corrélation avec une plus grande activation dans les basses fréquences thêta et delta. Bien sûr, comme dans la première étude pilote ces constats préliminaires devront être répliqués avec d'autres sujets.

\section{Conclusion}

Maintenant, où cela nous mène-t-il? Quelle pertinence présente ce type d'études pour les sciences de l'éducation. II a fallu une extraordinaire somme de travail de croisement de données pour se rendre jusque là, et, pourtant, la surface n'a été qu'effleurée. II reste à n'en pas douter un très long voyage à parcourir. Ceci étant dit, ces études pilotes ont permis d'analyser le comportement électro-physiologique du cerveau et du corps et de constater que ceux-ci s'activent différemment en fonction de la perception et du raisonnement face à la présentation de figures géométriques, et ce, de manière significative, tant qualitativement que statistiquement. II faut noter que ces résultats préliminaires sont présentés à titre d'illustration seulement 
et on ne peut pas généraliser à partir de ces derniers. Ils devront être complétés avec l'ensemble des données disponibles pour ce sujet et répliqués avec d'autres sujets.

Ultimement, ce type de recherche permettra une meilleure compréhension des processus cognitifs associés à l'apprentissage, l'enseignement et l'évaluation de figures géométriques dans l'enseignement des mathématiques. L'étude des relations entre les comportements externes et les comportements physiologiques plus cachés qui sont enregistrés en utilisant l'EEG peut favoriser l'émergence de nouvelles théories de la perception ou du raisonnement en mathématiques; ou aider à réfuter ou soutenir des modèles théoriques existants. II y a ici des implications évidentes pour les éducateurs de mathématiques qui veulent baser leur pédagogie sur des théories de la cognition et de l'apprentissage qui soient empiriquement appuyées. Ainsi, les données psychophysiologiques qui découlent par exemple de l'utilisation de l'EEG, l'EOG et l'EKG peuvent aider à départager des différences contradictoires entre: des données comportementales et verbales (discours phénoménologique); des théories cognitives; des interventions éducatives (Campbell, 2010; Goswami, 2006).

Bien sûr, ce type de recherche en est encore dans ses premiers balbutiements. S'agissant des applications directes dans la classe, il faut se rappeler qu'il demeure un écart important entre les expériences décrites dans cet article, ou ailleurs, et des indications précises pour l'enseignement (Ansari et Coch, 2006). À cet égard, la prudence est de mise, afin de ne pas participer à l'émergence de neuromythes qui font leur chemin jusque dans la classe (Geake, 2005; Goswani, 2006; Sousa, 2011). Comme le soutiennent Ferrari et McBride (2011), il est possible de généraliser aux neurosciences cognitive et sociale les remarques de William James (1899) qui, s'adressant à des enseignants, rappelle qu'ils commettent une erreur s'ils pensent que la psychologie en tant que science de l'esprit permet de concevoir directement des programmes et des méthodes d'apprentissage pour un usage immédiat dans la classe. Ces préoccupations clairement énoncées, il n'en demeure pas moins que le déploiement progressif et prudent de la recherche en neuroscience éducationnelle aura une importance cruciale pour l'éducation au XXIième siècle (Fischer, 2009, 2011), notamment pour l'enseignement des mathématiques (Campbell, 2010).

En ce qui concerne les recherches futures, les réponses de l'apprenant face à l'anxiété, et durant les moments de prise de conscience de concepts mathématiques, s'avèrent des phénomènes corporellement inscrits et constituent ainsi des candidats potentiels pour des études en neuroscience éducationnelle. II en est de même pour la résolution de problèmes 
individuelle et conjointe (voir les études sur la synchronisation entre cerveaux, par exemple celle de Dumas, Nadel, Soussignan, Martinerie et Garnero [2010]), pour l'ingénierie de l'espace d'apprentissage, spécialement celle impliquant l'utilisation d'ordinateurs et de programmes éducationnels basée sur l'interface cerveau-ordinateur (par exemple voir les programmes Fast ForWord et Earobic basés sur les recherches de Shaywitz [2003]). Tous ces thèmes de recherche présentent un intérêt potentiel pour l'étude empirique à partir d'une perspective radicale de l'inscription corporelle de l'esprit, de la cognition et de l'apprentissage.

La recherche en neuroscience éducationnelle s'avère un terrain transdisciplinaire riche qui offre une opportunité pour s'attaquer simultanément et de façon coordonnée, à la fois à des problèmes d'ordre philosophique et éducationnel, notamment à la nature et au développement de l'esprit-corps-cerveau. Elle permet de réconcilier à la fois les méthodes introspectives et béhaviorales (Campbell, 2011), en invoquant la perspective de l'inscription corporelle radicale de l'esprit (Thompson et Varela, 2001) qui requiert d'étudier l'être phénoménologique dans ses manifestations corporelles.

Ces études préliminaires illustrent que les frontières de la recherche en sciences de l'éducation peuvent encore s'étendre de façon à incorporer les méthodologies et techniques provenant d'autres disciplines, notamment les neurosciences cognitive et sociale; et que l'utilisation des techniques comme l'EEG est une façon de le faire (Makeig, 2010). La démarche présentée dans cet article suggère d'abord que ceci est possible et que des distinctions qualitatives et quantitatives, relatives aux processus cognitifs des apprenants, peuvent être faites en utilisant ces nouvelles méthodes et techniques. Des études supplémentaires faisant appel à différents niveaux d'analyse de l'objet esprit-corps-cerveau sont essentielles afin d'améliorer notre compréhension des processus impliqués dans l'apprentissage et ainsi favoriser ultimement des innovations didactico-pédagogiques dans l'enseignement.

\section{Références}

Ansari, D. et Coch, D. (2006). Bridges over troubled waters: Education and cognitive neuroscience. Trends in Cognitive Sciences, 10(4), 146-151. https://doi.org/10.1016/j.tics.2006.02.007 
Bartholow, B. D. et Amodio, D. M. (2009). Using event-related brains potentials in social psychological research: A brief review and tutorial. Dans E. Harmon-Jones et J. S. Beer (dir.), Methods in Social Neuroscience (p. 198-232). New-York, NY: The Guilford Press.

Bastiaansen, M. C. M., van Berkum, J. J. A. et Hagoort, P. (2002). Syntactic processing modulates the rhythm of the human EEG. Neurolmage, 17(3), 1479-1492. https://doi.org/10.1006/nimg.2002.1275

Bruer, J. T. (1997). Education and the brain: A bridge too far. Educational Researcher, 26(8), 4-16. https://doi.org/10.2307/1176301

Byrnes, J. P. (2001). Minds, brains, and learning. Understanding the psychological and educational relevance of neuroscientific research. New York, NY: The Guilford Press.

Campbell, S. R. (2011). Educational neuroscience: Motivation, methodology, and implications. Educational Philosophy and Theory, 43(1), 7-16. https://doi.org/10.1111/j.1469-5812.2010.00701.x

Campbell, S. R. (2010). Embodied minds and dancing brains: New opportunities for research in mathematics education. Dans B. Sriraman et L. English (dir.), Theories of mathematics education: Seeking new frontiers (p. 309-331). Berlin, Germany: Springer. https://doi.org/10.1007/978-3-642-00742-2 31

Campbell, S. R. (2004). Forward and inverse modelling: Mathematics at the nexus between mind and world. Dans H.-W. Henn et W. Blum (dir.), ICMI study 14: Applications and modelling in mathematics EducationPre-conference volume (p. 59-64). Dortmund, Germany: University of Dortmund.

Campbell, S. R. (2003). Reconnecting mind and world: Enacting a (new) way of life. Dans S. J. Lamon, W. A. Parker et S. K. Houston (dir.), Mathematical modelling: A way of life (p. 245-253). Chichester, UK: Horwood Publishing. https://doi.org/10.1533/9780857099549.5.245

Campbell, S. R. (2001). Enacting possible worlds: Making sense of (human) nature. Dans J. F. Matos, W. Blum, S. K. Houston et S. P. Carreira (dir.), Modelling and mathematics education (p. 3-14). Chichester, UK: Horwood Publishing. https://doi.org/10.1533/9780857099655.1.1 
Campbell, S. R. et Dawson, A. J. (1995). Learning as embodied action. Dans R. Sutherland et J. Mason (dir.), NATO advanced research workshop: Exploiting mental imagery with computers in mathematics education, NATO ASI Series F, vol. 138 (p. 233-249). Berlin, Germany: Springer. https://doi.org/10.1007/978-3-642-57771-0 16

Campbell, S. R., Handscomb, K., Zaparyniuk, N. E., Sha, L., Cimen, O. A. et Shipulina, O. V. (2009). Investigating image-based perception and reasoning in geometry. Paper presented to the American Educational Research Association: Brain, Neuroscience, and Education SIG (San Diego, CA). (ED505740). http://files.eric.ed.gov/fulltext/ED505740.pdf

Chalmers, D. (1998). The problems of consciousness. Dans H. Jasper, L. Descarries, V. Castellucci et S. Rossignol (dir.), Consciousness at the frontiers of neuroscience (Advances in neurology; Vol. 77). LippincottRaven Press.

Churchland, P. C. (1980). A perspective on mind-brain research. The journal of philosophy, 77(4), 185-207. https://doi.org/10.2307/2025588

Decety, J. (2011). Dissecting the neural mechanisms mediating empathy. Emotion Review, 3(1), 92-108. https://doi.org/10.1177/1754073910374662

Dehaene, S., Izard, V., Pica, P. et Spelke, E. (2006). Core knowledge of geometry in an Amazonian indigene group. Science, 311(5759), 381384. https://doi.org/10.1126/science.1121739

Descartes, R. (1996). Descartes: Meditations on first philosophy. With selections from the objections and replies (J. Cottingham, Ed.). (Original published in 1649). Cambridge, UK: Cambridge University Press. https://doi.org/10.1017/CBO9780511805028

Deschenaux, F. et Laflamme, C. (2007). Analyse du champ de la recherche en sciences de l'éducation au regard des méthodes qui y sont employées: la bataille est-elle vraiment gagnée pour le qualitatif? Recherches qualitatives, 27(2), 5-27.

Dumas, G., Nadel, J., Soussignan, R., Martinerie, J. et Garnero, L. (2010). Inter-brain synchronization during social interaction. PLoS One, 5(8), e12166. https://doi.org/10.1371/journal.pone.0012166

Ferrari, M. et McBride, H. (2011). Mind, brain, and education: The birth of a new science. Learning Landscapes, 5(1), 85-100.

http://ojs.learnquebec.ca/index.php/learnland/article/view/533 
Fischer, K. W. (2011). Dynamic cycles of cognitive and brain development: Measuring growth in mind, brain, and education. Dans, A. M. Battro, K. W. Fischer, et P. J. Léna (dir.), The Educated Brain. Essays in Neuroeducation (p. 127-150). New-York: Cambridge University Press. https://doi.org/10.1017/cbo9780511489907.010

Fischer, K. W. (2009). Mind, brain, and education: Building a scientific groundwork for learning and teaching. Mind, Brain, and Education, 3(1), 3-16. https://doi.org/10.1111/j.1751-228x.2008.01048.x

Fogarty, C. et Stern, J. A. (1989). Eye movement and blinks: Their relationship to higher cognitive processes. International Journal of Psychophysiology, 8(1), 35-42. https://doi.org/10.1016/01678760(89)90017-2

Gaetz, M., Weinberg, H., Rzempoluck, E. et Jantzen, K. J. (1989). Neural network classifications and correlation analysis of EEG and MEG activity accompanying spontaneous reversals of the Necker cube. Cognitive Brain Research, 6(4), 335-346. https://doi.org/10.1016/s0926-6410(97)00038-4

Geake, J. G. (2005). The neurological basis of intelligence: A contrast with "brain-based" education. Paper presented at the British Educational Research Association Annual Conference (Glamorgan, UK, September). http://www.leeds.ac.uk/educol/documents/156074.htm

Goswami, U. (2006). Neuroscience and education: from research to practice? Nature Reviews Neuroscience, 7(5), 406-413. https://doi.org/10.1038/nrn1907

Grech, R., Cassar, T., Muscat, J., Camilleri, K. P., Fabri, S. G., Zervakis, M., ... et Vanrumste, B. (2008). Review on solving the inverse problem in EEG source analysis. Journal of NeuroEngineering and Rehabilitation, 5(25), 1-33. https://doi.org/10.1186/1743-0003-5-25

Handscomb, K. (2007). Enactive cognition and Spinoza's theory of mind. Paper presented at the annual meeting of the North American Chapter of the International Group for the Psychology of Mathematics Education, University of Nevada, Reno, Nevada. http://citation.allacademic.com/meta/p193673 index.html

Harmon-Jones, E. et Peterson, C. K. (2009). Electroencephalographic methods in social and personality psychology. Dans E. Harmon-Jones et J. S. Beer (dir.), Methods in Social Neuroscience (p. 170-197). NewYork: The Guilford Press. 
Immordino-Yang, M. H. (2011). Implications of affective and social neuroscience for educational theory. Educational Philosophy and Theory, 43(1), 98-103. https://doi.org/10.1111/j.1469-5812.2010.00713.x

James, W. (1899). Talks to teachers on psychology and to students on some of life's ideals. https://doi.org/10.1037/10814-000

Just, M. A., Carpenter, P. A. et Miyake, A. (2003). Neuroindices of cognitive workload: Neuroimaging, pupillometric and event-related potential studies of brain work. Theoretical Issues in Ergonomics Science, 4(12), 56-88. https://doi.org/10.1080/14639220210159735

Knyazev, G. G., Savostyanov, A. N. et Levin, E. A. (2005). Alpha synchronization and anxiety: Implications for inhibition vs. alertness hypotheses. International Journal of Psychophysiology, 59(2), 151158. https://doi.org/10.1016/j.ijpsycho.2005.03.025

Kramer, A. F. et McCarley, J. S. (2003). Oculomotor behaviour as a reflection of attention and memory processes: Neural mechanisms and applications to human factors. Theoretical Issues in Ergonomics Science, 4(1-2), 21-55. https://doi.org/10.1080/14639220210159744

Kuhn, T. S. (1962). La structure des révolutions scientifiques (Traduit par L. Meyer). Paris: Flammarion.

Lee, K., Lim, Z. Y., Yeong, S. H. M., Ng, S. F., Venkatraman, V. et Chee, M. W. L. (2007). Strategic differences in algebraic problem solving: Neuroanatomical correlates. Brain Research, 1155, 163-171. https://doi.org/10.1016/j.brainres.2007.04.040

Lenroot, R. K. et Giedd, J. N. (2006). Brain development in children and adolescents: Insights from anatomical magnetic resonance imaging. Neuroscience \& Biobehavioral Reviews, 30(6), 718-729. https://doi.org/10.1016/j.neubiorev.2006.06.001

Lutz, A., Lachaux, J.-P., Martinerie, J. et Varela, F. J. (2002). Guiding the study of brain dynamics by using first-person data: Synchrony patterns correlate with ongoing conscious states during a simple visual task. PNAS, 99(3), 1586-1591. https://doi.org/10.1073/pnas.032658199

Makeig, S. (2010). Commentary on embodied minds and dancing brains: New opportunities for research in mathematics education. Dans B. Sriraman et I. English (dir.), Theories of mathematics education: Seeking new frontiers (p. 333-337). Berlin: Springer. https://doi.org/10.1007/978-3-642-00742-2 32 
Makeig, S., Bell, A. J., Jung, T.-P. et Sejnowski, T. J. (1996). Independent component analysis of electroencephalographic data. Dans D. Touretzky, M. Mozer et M. Hasselmo (dir.), Advances in Neural Information Processing Systems 8 (p. 145-151). Cambridge, MA: MIT Press.

https://papers.nips.cc/paper/1091-independent-component-analysis-ofelectroencephalographic-data.pdf

Malmivuo, J. et Plonsey, R. (1995). Bioelectromagnetism: Principles and applications of bioelectric and biomagnetic fields. New York, NY: Oxford University Press. https://doi.org/10.1093/acprof:oso/9780195058239.001.0001

Merleau-Ponty, M. (2010). Oeuvres. France: Éditions Gallimard.

Merleau-Ponty, M. (1962). Phenomenology of perception. London, UK: Routledge and Kegan Paul.

Michel, C. M., Lantz, G., Spinelli, L., De Peralta, R. G., Landis, T. et Seeck, M. (2004). 128-channel EEG source imaging in epilepsy: Clinical yield and localization precision. Journal of Clinical Neurophysiology, 21(2), 71-83. https://doi.org/10.1097/00004691-200403000-00001

Pagé, P., Strayer, F. F. et Reid, L. (2001). Où en est la cognition sociale? Sociogenèse et sélection ontogénétique des pensées sociales. Psychologie canadienne, 42(3), 185-199. https://doi.org/10.1037/h0086891

Patten, K. E. et Campbell, S. R. (2011). Introduction: Educational Neuroscience. Educational Philosophy and Theory, 43(1), 1-6. https://doi.org/10.1111/j.1469-5812.2010.00700.x

Pitts, M. A., Martinez, A., Stalmaster, C., Nerger, J. L. et Hillyard S. A. (2009). Neural generators of ERPs linked with Necker cube reversals. Psychophysiology, 46(4), 694-702. https://doi.org/10.1111/j.14698986.2009.00822.x

Scherg, M. (1990). Fundamentals of dipole source potential analysis. Dans F. Grandori, M. Hoke et G. L. Romani (dir.), Auditory evoked magnetic fields and electric potentials - Advances in audiology, Vol. 6 (p. 40-69). Karger: Basel.

Shaywitz, S. (2003). Overcoming dyslexia: A new and complete sciencebased program for reading problems at any level. New-York, NY: Alfred A. Knopf, a division of Random House. 
Shimaoka, D., Kitajo, K., Kaneko, K. et Yamaguchi, Y. (2010). Transient process of cortical activity during Necker cube perception : From local clusters to global synchrony. Nonlinear Biomedical Physics, 4(suppl. 1), S7. https://doi.org/10.1186/1753-4631-4-s1-s7

Snook, I. (2012), Educational Neuroscience: A plea for radical scepticism. Educational Philosophy and Theory, 44(5), 445-449. https://doi.org/10.1111/j.1469-5812.2011.00831.x

Sousa, D. A. (2011). Mind, brain, and education: The impact of educational neuroscience on the science of teaching. Learning Landscapes, 5(1), 37-43. http://ojs.learnquebec.ca/index.php/learnland/article/view/529

Steinberg, L. (2007). Risk taking in adolescence: New perspectives from brain and behavioral science. Current Directions in Psychological Science, 16(2), 55-59. https://doi.org/10.1111/j.1467-8721.2007.00475.x

Thompson, E. (2007). Mind in life: Biology, phenomenology and the sciences of mind. Boston, MA: Harvard University Press.

Thompson, E. et Varela, F. J. (2001). Radical embodiment: neural dynamics and consciousness. Trends in Cognitive Sciences, 5(10), 418-425. https://doi.org/10.1016/s1364-6613(00)01750-2

Tochon, F. V. (1996). Rappel stimulé, objectivation clinique, réflexion partagée. Fondements méthodologiques et applications pratiques de la rétroaction vidéo en recherche et formation. Revue des sciences de l'éducation, 22(3), 467-502. https://doi.org/10.7202/031889ar

Turner, D. A. (2011). Which part of 'two way street' did you not understand? Redressing the balance of neuroscience and education. Educational Research Review, 6(3), 223-231. https://doi.org/10.1016/j.edurev.2011.10.002

Vaisanen, O. (2008). Multichannel EEG methods to improve the spatial resolution of cortical potential distribution and the signal quality of deep brain sources. Thèse de doctorat inédite. Tampere University of Technology. https://pdfs.semanticscholar.org/8808/29671dd1e717829dc612f0561d 9a61e0e9df.pdf

Van Leijenhorst, L., Moor, B. G., Op de Macks, Z. A., Rombouts, S. A. R. B., Westenberg, P. M. et Crone, E. A. (2010). Adolescent risky decisionmaking: Neurocognitive development of reward and control regions. Neurolmage, 51(1), 345-355. 
https://doi.org/10.1016/j.neuroimage.2010.02.038

Varela, F. J. et Shear, J. (1999). First-person accounts: Why, what, and how. Dans F. J. Varela et J. Shear (dir.), The view from within: First-person approaches to the study of consciousness (p. 1-14). Exeter, UK: Imprint Academic.

Varela, F. J., Thompson, E. et Rosch, E. (1991). The embodied mind: Cognitive science and human experience. Cambridge, MA: MIT Press.

Vermersch, P. (1994). L'entretien d'explicitation en formation initiale et en formation continue. Paris : ESF.

Vygotski, L. S. (1978). Mind in society. London, UK: Harvard University Press.

Winegar, L. T. (1996). Developmental research and comparative perspectives : Applications to developmental science. Dans J. Tudge, M. J. Shanahan et J. Valsiner, (dir.), Comparisons in Human Development. Understanding Time and Context (p.13-33). Cambridge, UK: Cambridge University Press. https://doi.org/10.1017/cbo9780511720345.002 